\title{
EFECTO DE CERAS COMO COMPLEMENTO A LA INMERSIÓN HIDROTÉRMICA SOBRE LA CALIDAD EN LA FRUTA DE PAPAYA (Carica papaya L. HÍBRIDO POCOCÍ)
}

\author{
V. Gustavo Corrales ${ }^{* *}$, Gerardina Umaña $a^{1 / *}$ \\ Palabras clave: Antracnosis; tasa respiratoria; pérdida de peso; poscosecha. \\ Keywords: Anthracnose; respiration rate; weight loss; postharvest. \\ Recibido: 01/10/14 \\ Aceptado: 11/12/14
}

\section{RESUMEN}

Se evaluó el efecto de ceras, como complemento a la inmersión hidrotérmica, sobre variables de calidad poscosecha de papaya. Las frutas se cosecharon, empacaron y fueron lavadas, desinfectadas e inmersas en agua a $49^{\circ} \mathrm{C}$ por 20 minutos (TH) para posteriormente aplicar los tratamientos: 1) cera de abejas y aceite de palma $5 \%$; 2) cera de mezcla ácidos grasos $4,7 \%$; 3 ) quitosano $0,1 \%$; 4) solo $\mathrm{TH} ; 5$ ) testigo (sin inmersión hidrotérmica ni cera). La papaya fue almacenada 15 días a $12^{\circ} \mathrm{C}$ y posteriormente a $20^{\circ} \mathrm{C}$. Se encontraron diferencias significativas $(\mathrm{p}<=0,05)$ entre las frutas que recibieron el TH complementado con cera de abejas y palma al 5\%, que tuvieron menor tasa respiratoria $(12,27 \mathrm{ml} \mathrm{CO} / \mathrm{kg} * \mathrm{~h}$ a 8 días de salida del almacenamiento en frío), en comparación con las que solo recibieron el TH $(16,72 \mathrm{ml} \mathrm{CO} / 2 / \mathrm{kg} * \mathrm{~h})$, o las papayas testigo (17,01 $\mathrm{ml} \mathrm{CO} / \mathrm{kg} * \mathrm{~h})$. El menor porcentaje de pérdida de peso acumulado se registró con el TH más cera de abejas y palma. Las variables de color no fueron afectadas, excepto por el tratamiento 2, cera de mezcla de ácidos grasos, que indujo un retraso en el desarrollo del color $(\mathrm{p} \leq 0,05)$. No se observaron cambios en firmeza interna o externa

\begin{abstract}
The effect of waxes, as a complement to hydrothermal immersion, on the quality of papaya fruit (Carica papaya L. Pococí hybrid). Different waxes were evaluated as a complement to hydrothermal treatment on the overall papaya fruit quality parameters. The fruits were harvested, washed in water, disinfected with sodium hypochlorite and exposed to hydrothermal immersion treatment at $49^{\circ} \mathrm{C} / 20$ min (HT); then treatments were applied: 1) bees wax +palm oil $5 \%$ solution; 2) fatty acids wax mixture $4.7 \%$; 3) chitosan $0.1 \%$; 4) only HT; 5) control (no hydrothermal immersion nor wax). The fruit was stored for 15 days at $12^{\circ} \mathrm{C}$, then at $20^{\circ} \mathrm{C}$. Significant differences $(\mathrm{p} \leq 0.05)$ were found between fruits receiving HT complemented with $5 \%$ bees + palm oil wax, which exhibited lower respiration rate $\left(12.27 \mathrm{ml} \mathrm{CO} / \mathrm{kg}^{*} \mathrm{~h}\right.$ a 8 days after leaving cold storage), as compared with those receiving only $\mathrm{HT}\left(16.72 \mathrm{ml} \mathrm{CO}_{2} /\right.$ $\mathrm{kg} * \mathrm{~h})$ or control fruits $(17.01 \mathrm{ml} \mathrm{CO} / \mathrm{kg} * \mathrm{~h})$. The lesser percent of acumulated weight loss was registered whit $\mathrm{TH}$ plus bees wax cund palm oil. The color parameters were not affected, except for treatment 2 , fatty acids wax mixture, which
\end{abstract}

\footnotetext{
1 Autor para correspondencia. Correo electrónico: gerardina.umana@ucr.ac.cr

Centro de Investigaciones Agronómicas, Universidad de Costa Rica. San José, Costa Rica.
} 
ni en grados brix. El TH permitió una disminución en la incidencia de pudriciones, severidad de antracnosis y prolongación de la vida útil. El uso de ceras como complemento al TH puede contribuir a mantener algunos de los parámetros que inciden en la calidad de la papaya.

\section{INTRODUCCIÓN}

La papaya (Carica papaya L.), es nativa de América Central y se considera la especie de mayor importancia económica de la familia Caricaceae. El cultivo ha tenido un auge en los últimos años en Costa Rica, asociado con la introducción del híbrido Pococí, lo que permitió exportar el equivalente a 4932,7 toneladas, correspondientes a US\$ 3889,3 mil (PROCOMER 2014). De acuerdo con la FAO (2013), la mayor limitante para exportar la fruta ha sido el manejo fitosanitario, por cuanto los importadores exigen el cumplimiento de una serie de requisitos y de seguridad alimentaria que condicionan la entrada de la papaya en sus mercados.

Dentro de las principales limitantes fitosanitarias se encuentra la antracnosis en papaya causada por el hongo Colletotrichum gloeosporioides Penz. Sacc. De acuerdo con algunos autores (Paull y Chen 1983, Protsenko et ál. 2010), el patógeno puede secretar la enzima poligalacturonasa que digiere componentes de la pared celular del fruto que favorece una serie de reacciones que originan alteraciones en los tejidos durante el proceso de maduración.

Como alternativas para el combate de enfermedades poscosecha, se han implementado prácticas innovadoras tales como los tratamientos hidrotérmicos, con los que se busca reducir induced a delay in color development $(\mathrm{p} \leq 0.05)$. No changes were observed in internal or external firmness, nor in degrees brix. HT reduced the incidence of peduncular rot and anthracnose severity $(\mathrm{p} \leq 0.05)$, and extended useful life time. The use of waxes as a complement to HT can contribute to preserving some of the parameters which influence the final papaya fruit quality.

la expresión de infecciones latentes de organismos (Bautista et ál. 2006, Fallik 2004), y han resultado ser tan eficaces como los tratamientos químicos para combatir enfermedades causadas por hongos y daños por plagas insectiles y que permite conservar la calidad de frutas y vegetales (Lurie 1998, Tang et ál. 2007).

La inmersión de frutas como papaya, litchi o carambola en agua caliente a $49^{\circ} \mathrm{C}$ por $20 \mathrm{~min}$ para combatir la mosca de la fruta, es un requisito aprobado en Estados Unidos por el USDAAPHIS (Fallik 2004) para que pueda ingresar producto obtenido en Hawaii al mercado estadounidense, y es una condición que Costa Rica debe de cumplir si exporta papaya a ese mercado (Saborío 2012).

La respuesta de los productos agrícolas a los tratamientos con calor está influenciada por la especie, el cultivar, las condiciones prevalecientes durante el desarrollo del cultivo en el campo, la madurez a la cosecha, el manejo entre la cosecha y la aplicación de los tratamientos y el método utilizado para la aplicación del calor (Lurie y Mitcham 2007). Vargas (2013) informó que la papaya híbrido Pococí que recibió tratamiento hidrotérmico $49^{\circ} \mathrm{C} / 20$ min presentó para algunas fechas de cosecha, bronceados a nivel de cáscara, permanencia de zonas verdes que no llegaron a madurar y una pérdida de peso mayor 
en comparación a la fruta del testigo. Lara et ál. (2014) indican que conforme se incrementa la temperatura de exposición al aplicar el tratamiento con calor sobre las frutas, ocurre un descenso de la fuerza y rigidez de la cutícula, dado el efecto de plasticidad que ejerce el agua sobre la cutícula.

Por su parte, la transpiración de las frutas en general durante la etapa poscosecha, favorecida por cambios en las condiciones de almacenamiento, puede acelerar la marchitez, el arrugamiento, la pérdida de firmeza, el peso y la apariencia, factores determinantes en el valor final del producto, sin omitir que una pérdida de peso del 3\% tiene implicaciones negativas en la apariencia del producto, restándole valor e inclusive rechazo en el mercado (Bartz y Bretch 2003).

Por consiguiente, ha surgido un interés en el uso de recubrimientos comestibles o ceras comerciales, que crean una barrera selectiva que modifica la transmisión de gases, vapor de agua y otros solutos lo que reduce la actividad metabólica con la consecuente disminución de la velocidad de transpiración y respiración, la pérdida de agua y retardo de la maduración lo que contribuye a prolongar la calidad.

La eficacia de las ceras en la reducción de la pérdida de peso está ligada a varios factores como la composición química, la dosis y cobertura lograda sobre el producto a la hora de la aplicación (Beiruth et ál. 2009, Valencia et ál. 2009). Aún cuando las ceras pueden mejorar la apariencia de los frutas, algunas pueden dejar residuos o una textura pegajosa (Bai y Plotto 2012, Bartz y Brecht 2003).

Las ceras son formuladas a partir de productos sintéticos o derivarse de productos naturales como la cera de abejas y carnauba, aceites vegetales y minerales. Algunas ceras o recubrimientos tienen como base polisacáridos como almidón, celulosa, quitosano, mientras que otras están hechas a partir de proteínas de maíz, trigo o soya, considerándose estas últimas como ceras comestibles (Bai y Plotto 2012).

De acuerdo con lo anterior, se planteó este estudio con el objetivo de evaluar el efecto de las ceras como complemento al tratamiento hidrotérmico de $49^{\circ} \mathrm{C}$ por 20 min sobre las variables de calidad en la papaya híbrido Pococí.

\section{MATERIALES Y MÉTODOS}

Se trabajó con frutas provenientes de fincas comerciales asociadas al proyecto productivo para mercado de exportación del Centro Agrícola Cantonal de Guácimo, ubicado en la provincia de Limón, Costa Rica, donde los agricultores de la zona administran el cultivo bajo un programa de manejo técnico elaborado y supervisado por dicho centro.

Se seleccionaron papayas del híbrido Pococí para lo que se siguieron los estándares de calidad que se aplican para la exportación se seleccionaron frutas recién cosechadas, sanas, sin daños, ni pudriciones aparentes, de tamaño similar, con un peso promedio de $1400 \mathrm{~g}$, estado de madurez entre 3 a 5 pintas o franjas de maduración, que en promedio abarcaban un 13\% de color amarillo, correspondiente al grado uno de la escala de maduración propuesta por Salazar (2012). Cada papaya se envolvió con papel periódico para evitar daños mecánicos, luego se colocó dentro de cajas plásticas con capacidad para 8 unidades de papaya, y fueron trasladadas cubiertas con una lona hasta el Laboratorio de Tecnología Poscosecha del Centro de Investigaciones Agronómicas de la Universidad de Costa Rica, ubicado en Sabanilla de Montes de Oca, provincia de San José donde tuvo lugar la fase de aplicación de tratamientos y las evaluaciones.

Las frutas fueron lavadas con agua, desinfectadas mediante una inmersión durante 60 segundos en solución de hipoclorito de sodio a $100 \mathrm{mg} . \mathrm{l}^{-1}$, luego se completó su secado a temperatura ambiente y se aleatorizaron e identificaron según el tratamiento.

\section{Tratamiento hidrotérmico (TH)}

El tratamiento hidrotérmico consistió en sumergir las frutas en agua a una temperatura controlada de $49^{\circ} \mathrm{C}$ por 20 min en un baño María, inmediatamente después de los cuales se procedió a asperjar agua a temperatura ambiente 
por 20 min para reducir la temperatura en las papayas. Posteriormente se hizo la inmersión de la fruta en los tratamientos, entre los que se incluyó una cera de ácidos grasos emulsionados de cera de abejas, y aceite de palma (Verdiol 111 (̊), una cera de mezcla de ácidos grasos de aceite vegetal de grado alimenticio, glicerol grado alimenticio, y sorbitán grado alimenticio (JBT 2981®), quitosano proporcionado por el Laboratorio de Polímeros (POLIUNA) de la Universidad Nacional, además de las papayas a los que no se les aplicó ningún tipo de recubrimiento, incluyéndose así un total de 5 tratamientos, según se detalla en el Cuadro 1.

Cuadro 1. Descripción de los tratamientos aplicados mediante inmersión de la papaya.

\begin{tabular}{|c|c|c|}
\hline \multicolumn{3}{|c|}{ Tratamientos } \\
\hline & & Dosis cera \\
\hline 1. Tratamiento hidrotérmico $49^{\circ} \mathrm{C}$ por $20 \mathrm{~min}(\mathrm{TH})$ y & cera de abejas y aceite de palma (Verdiol 11L $®$ ) & $5,0 \%$ \\
\hline 2. Tratamiento hidrotérmico $49^{\circ} \mathrm{C}$ por $20 \mathrm{~min}(\mathrm{TH})$ y & cera de mezcla de ácidos grasos (JBT 2981®) & $4,7 \%$ \\
\hline 3. Tratamiento hidrotérmico $49^{\circ} \mathrm{C}$ por $20 \mathrm{~min}(\mathrm{TH})$ y & quitosano & $0,1 \% *$ \\
\hline 4. Tratamiento hidrotérmico $49^{\circ} \mathrm{C}$ por $20 \mathrm{~min}(\mathrm{TH})$ & sin cera & - \\
\hline 5. Testigo (sin tratamiento térmico y sin cera) & & - \\
\hline
\end{tabular}

*Quitosano disuelto en ácido acético al 0,5\% (m/v).

Inmediatamente, cada fruta se envolvió hasta la mitad con papel periódico blanco incluyendo la zona peduncular y media y se colocó de manera vertical en cajas plásticas y dentro de una cámara de frío a $12^{\circ} \mathrm{C} \pm 2^{\circ} \mathrm{C}$ (controlador digital Johnson Controls Penn $\left.{ }^{\circledR}\right)$, con un ámbito de humedad relativa entre $85-90 \%$, durante 15 días. Pasado este periodo la temperatura de almacenamiento se incrementó a $20^{\circ} \mathrm{C}$ con $90 \%$ de humedad, lo que se denomina como almacenamiento a temperatura ambiente hasta que la fruta alcanzara el estado de maduración para consumo.

Con el objetivo de determinar la calidad inicial de la papaya, se tomaron 8 frutas al azar para analizar las propiedades fisicoquímicas, color y tasa respiratoria antes de su ingreso a cámara a $12^{\circ} \mathrm{C}$. Las evaluaciones se hicieron: el día de entrada almacenamiento a $12^{\circ} \mathrm{C}$, durante el almacenamiento a $12^{\circ} \mathrm{C}$, día de la salida de almacenamiento en frío (dsaf), 4 días de salida de almacenamiento en frío ( 4 dsaf), 8 días de salida de almacenamiento en frío ( 8 dsaf) y 12 días de salida de almacenamiento en frío (12 dsaf).
Las variables evaluadas fueron la tasa respiratoria $\left(\mathrm{ml} \cdot \mathrm{kg}^{-1} \cdot \mathrm{h}^{-1}\right.$ de $\left.\mathrm{CO}_{2}\right)$, para lo que se colocó la fruta en un recipiente a manera de sistema cerrado donde se mantuvo por $3 \mathrm{~h}$ antes de efectuar la medición. Cada lectura consistió en muestrear aire interno del recipiente durante $15 \mathrm{~s}$, al final se le insertó el lector de dióxido de carbono de un analizador portátil de gases Bridge Analyzer Inc ®. A partir de las lecturas, se determinó la tasa respiratoria según la fórmula que se muestra a continuación (Umaña et ál. 2011):

$$
\mathrm{CO}_{2}\left(\mathrm{ml}^{\mathrm{kg}} \mathrm{kg}^{-1} \cdot \mathrm{h}^{-1}\right)=\frac{\left[\mathrm{CO}_{2} \mathrm{f}\right]-\left[\mathrm{CO}_{2} \mathrm{i}\right]}{100} \times \frac{\text { Vrep }-\mathrm{P}}{\frac{\mathrm{P} \times \mathrm{T}}{1000}}
$$

Donde:

$\left[\mathrm{CO}_{2} \mathrm{f}\right]=$ Concentración final de $\mathrm{CO}_{2}(\%)$

$\left[\mathrm{CO}_{2} \mathrm{i}\right]=$ Concentración inicial de $\mathrm{CO}_{2}(\%)$

Vrep= Volumen del recipiente en $\mathrm{cm}^{3}$

$\mathrm{P}=$ Peso del fruto en gramos

$\mathrm{T}=$ Tiempo en horas 
Se evaluó la pérdida de peso (\%) para lo cual se utilizó el método de Acosta et ál. (2001), el color externo e interno fue medido visualmente como porcentaje de avance del color amarillo, para el desarrollo del color se utilizó un colorímetro electrónico Minolta CR-300 series obteniéndose los valores de $\mathrm{L}^{*}, \mathrm{a}^{*} \mathrm{y} \mathrm{b}^{*}$ con los cuales se obtuvieron el hue $\left(h^{\circ}\right)$ y chroma $\left(C^{*}\right)$, la firmeza se midió con un penetrómetro Chatillon 1M DPP-100 y se anotó la fuerza de penetración (Newtons) requerida por un puntal en forma de cincel en 3 puntos del exocarpo localizados en la zona ecuatorial de la cáscara $(1 \mathrm{~mm}$ de grosor), para registrar el valor en el cual la cáscara cedió a la fuerza aplicada. Para la medición de la firmeza en la pulpa, se eliminó el tejido comprendido por el exocarpo del fruto muy cerca de los 3 puntos donde se midió la firmeza de la cáscara pero separados, y se procedió a medir la firmeza registrada hasta una profundidad entre 5 a $10 \mathrm{~mm}$. Para obtener los grados brix (\%) se tomaron las muestras en la zona ecuatorial de cada fruto, para lo cual se eliminaron la cáscara y las semillas para luego proceder a licuar la pulpa y medir los grados brix del filtrado de pulpa por medio de un refractómetro digital marca Atago PR 100.

Para determinar la incidencia de antracnosis y pudrición peduncular, se contabilizó el número de frutas afectadas del total por repetición para obtener el porcentaje de frutas afectadas. La severidad de pudriciones se midió como el porcentaje de área afectada por lesiones de antracnosis a partir de la guía sobre la escala de severidad propuesta por Arauz y Navarro (1999). Se determinó el área bajo la curva de progreso de la enfermedad, mediante la siguiente ecuación (González et ál. 1999, modificado):

$$
A B C P E=\left(\underline{l}_{1}+I_{2}\right) \times h_{1}+\left(l_{2}+I_{3}\right) \times h_{2}+\left(l_{n}+I_{n}\right) \times h_{n}
$$

2

Donde:

$\mathrm{A}=$ área bajo la curva del desarrollo de la enfermedad.

$\mathrm{I}_{1}=$ el valor de la incidencia en la primera fecha de evaluación.

$\mathrm{I}_{2}=$ el valor de la incidencia en la segunda fecha de evaluación.

$\mathrm{h}_{1}=$ el valor del tiempo en días transcurridos entre la primera fecha y la segunda fecha de evaluación. $\mathrm{I}_{3}=$ el valor de la incidencia en la tercera fecha de evaluación.

$\mathrm{h}_{2}=$ el valor del tiempo en días transcurridos entre la segunda y la tercera fecha de evaluación.

$\mathrm{I}_{\mathrm{n}}=$ el valor de la incidencia en $n$ fechas de evaluación.

$\mathrm{h}_{\mathrm{n}}=$ el valor del tiempo en días trascurridos entre $n$ fechas de evaluación.

Se utilizó un diseño experimental irrestricto al azar con 5 repeticiones por tratamiento, con 6 frutas por repetición para un total de 30 frutas por tratamiento. A los datos se les aplicó un análisis de varianza (ANOVA), la separación de medias de los tratamientos se realizó mediante la prueba LSD de Fisher ( $\mathrm{p} \leq 0,05)$.

\section{RESULTADOS Y DISCUSIÓN}

La tasa respiratoria de la papaya varió durante las diferentes evaluaciones. Luego de aplicados los tratamientos y antes de ingresar la fruta al almacenamiento en frío a $12^{\circ} \mathrm{C}$ (Figura 1), la tasa respiratoria se redujo en un $59 \%$ en las frutas con tratamiento hidrotérmico (TH) y cera de abeja y aceite de palma, y en un $40 \%$ en las papayas con tratamiento hidrotérmico (TH) y cera de mezcla de ácidos grasos, en comparación con las frutas del testigo y tratamiento hidrotérmico $(\mathrm{TH})$ sin cera.

En la medición que se hizo a los 14 días de almacenamiento mientras la fruta se mantenía 


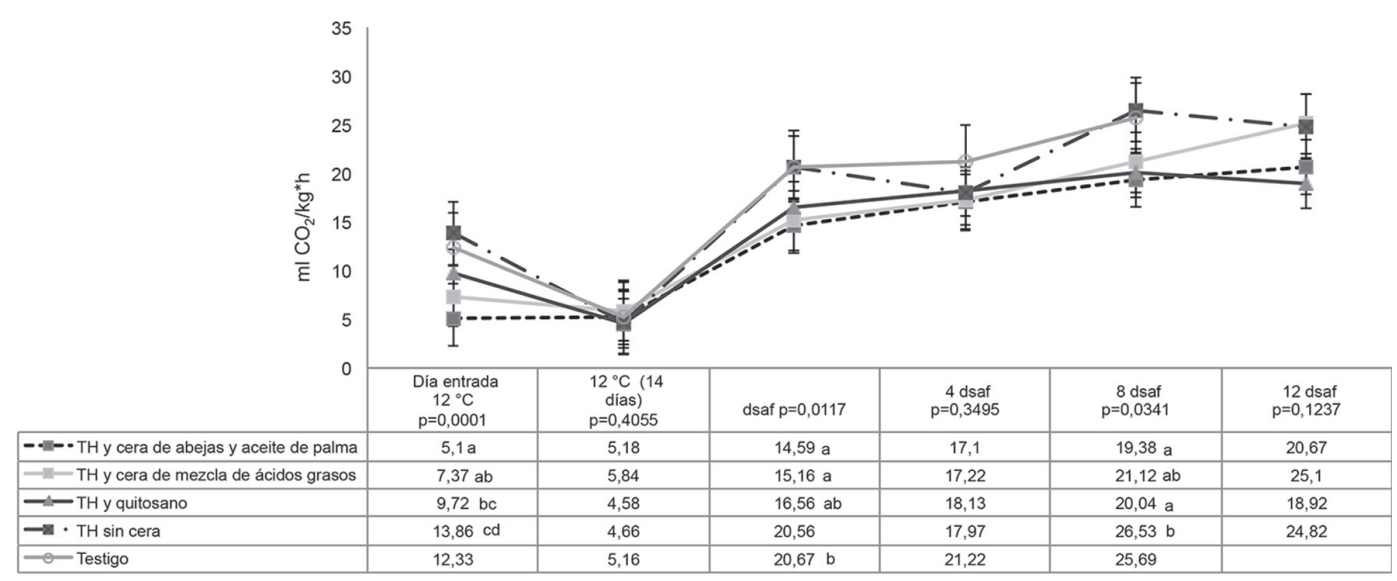

Fig. 1. Respiración de frutas de papaya con tratamiento hidrotérmico $49^{\circ} \mathrm{C} / 20 \mathrm{~min}(\mathrm{TH})$ y ceras. Letras distintas en columnas indican diferencias significativas según prueba LSD Fisher $(\mathrm{p}<=0,05)$. Barras indican error estándar.

a $12^{\circ} \mathrm{C}$, la tasa respiratoria fue baja, básicamente por el efecto del frío y no se detectaron diferencias entre las papayas de los diferentes tratamientos. El día que la fruta salió del almacenamiento en frío (dsaf) y se aumentó a $20^{\circ} \mathrm{C}$ la temperatura de almacenamiento, hubo un incremento significativo en la respiración, con valores comprendidos entre $14,5 \mathrm{ml} \cdot \mathrm{kg}^{-1} \cdot \mathrm{h}^{-1}$ de $\mathrm{CO}_{2}$ y $20,67 \mathrm{ml} \cdot \mathrm{kg}^{-1} \cdot \mathrm{h}^{-1}$ de $\mathrm{CO}_{2}$, evaluación en la cual las frutas con el tratamiento hidrotérmico complementado con cera de abejas y aceite de palma o la cera mezcla de ácidos grasos presentaron tasas significativamente menores con respecto a la papaya con el tratamiento hidrotérmico $(\mathrm{TH})$ sin cera y la del testigo. En el periodo en que la fruta se mantuvo almacenada a $20^{\circ} \mathrm{C}$, se observó una tendencia donde las papayas de los tratamientos testigo y tratamiento hidrotérmico $(\mathrm{TH})$ sin cera fueron las que tuvieron la mayor tasa de respiración, sin diferencias estadísticas entre las frutas de esos 2 tratamientos. El pico en la tasa respiratoria se presentó a los 8 dsaf, registrándose los menores valores de $\mathrm{CO}_{2}$ en la fruta tratada con cera de abejas y aceite de palma, seguida de la del tratamiento con quitosano $0,1 \%$, mientras que las frutas con tratamiento hidrotérmico (TH) sin cera y las del testigo presentaron los mayores valores, $26,53 \mathrm{y}$ 25,69 $\mathrm{ml} . \mathrm{kg}^{-1} \cdot \mathrm{h}^{-1}$ de $\mathrm{CO}_{2}$ respectivamente.
Los resultados obtenidos en este ensayo coinciden con lo señalado por Castricini (2009), en papaya Golden, donde las frutas del testigo exhibieron los mayores valores en la tasa respiratoria con $22 \mathrm{ml} \cdot \mathrm{kg}^{-1} \cdot \mathrm{h}^{-1}$ de $\mathrm{CO}_{2}$ en promedio, para superar hasta en un $50 \%$ a la papaya tratada en esa investigación con almidón de yuca al $3 \%$. Según dicho autor, lo obtenido en su trabajo se relacionó con el grado de espesor que formó la cobertura del tratamiento sobre el exocarpo de la fruta y que permitió una importante reducción de la intensidad de respiración.

La papaya perdió peso durante el almacenamiento (Cuadro 2). El día de salida del almacenamiento en frío (dsaf), se encontró que las frutas tratadas con el tratamiento térmico (TH) y cera de abejas y aceite de palma y las del tratamiento térmico $(\mathrm{TH})$ sin encerado, tuvieron menor pérdida de peso con respecto a las de los tratamientos térmico $(\mathrm{TH})$ con quitosano al $0,1 \%$, tratamiento térmico $(\mathrm{TH})$ con cera de mezcla de ácidos grasos y testigo. A los 4 dsaf, nuevamente la pérdida de peso fue menor en la fruta con tratamiento térmico complementado con cera de abejas y aceite de palma, y la del tratamiento térmico (TH) sin encerado, sin diferencias estadísticas con las papayas con tratamiento térmico (TH) y cera de mezcla de ácidos grasos. En esta 
Cuadro 2. Pérdida de peso en frutas de papaya con tratamiento hidrotérmico $49^{\circ} \mathrm{C} / 20 \mathrm{~min}(\mathrm{TH})$ y ceras.

\begin{tabular}{lccc}
\hline \multicolumn{1}{c}{ Tratamiento } & \multicolumn{3}{c}{$\begin{array}{c}\text { Porcentaje de pérdida de peso } \\
\text { Evaluaciones* }\end{array}$} \\
\hline & \multicolumn{1}{c}{ dsaf } & 4 dsaf & Acumulado \\
\cline { 2 - 4 } TH y cera de abejas y aceite de palma & $1,31 \mathrm{a}$ & $0,53 \mathrm{a}$ & $1,86 \mathrm{a}$ \\
TH y cera de mezcla de ácidos grasos & $1,50 \mathrm{c}$ & $0,60 \mathrm{ab}$ & $2,04 \mathrm{ab}$ \\
TH y quitosano & $1,57 \mathrm{c}$ & $0,78 \mathrm{bc}$ & $2,31 \mathrm{c}$ \\
TH sin cera & $1,37 \mathrm{ab}$ & $0,70 \mathrm{ab}$ & $2,24 \mathrm{bc}$ \\
Testigo & $1,46 \mathrm{bc}$ & $0,93 \mathrm{c}$ & $2,29 \mathrm{bc}$ \\
\hline$p$-valor & 0,0003 & 0,0058 & 0,0036 \\
\hline
\end{tabular}

*Letras distintas en columnas indican diferencias significativas según prueba LSD Fisher $(\mathrm{p}<=0,05)$.

fecha de evaluación, la mayor pérdida de peso se encontró en la fruta testigo.

La pérdida de peso acumulada durante las observaciones alcanzó un 1,86\% en la fruta con tratamiento térmico $(\mathrm{TH})$ y con cera de abejas y aceite de palma, y un 2,04\% en la fruta con tratamiento térmico $(\mathrm{TH})$ y cera de mezcla de ácidos grasos, sin diferencias estadísticas entre estos tratamientos $(\mathrm{p}<=0,05$ según prueba LSD Fisher). Un segundo grupo estuvo constituido por las papayas que perdieron más peso; las mismas correspondieron a las del tratamiento térmico (TH) complementado con quitosano (2,31\%), las del tratamiento térmico (TH) sin encerar $(2,24 \%)$ y las papayas del tratamiento testigo $(2,29 \%)$, mostrándose una tendencia en la disminución de la pérdida de peso que ejercen los tratamientos complementarios con las ceras, y que permiten percibir la fruta con mejor apariencia.

En investigaciones efectuadas por Bautista et ál. (2003) en las que evaluaron el quitosano en concentraciones de $0,1 \%$ y $0,5 \%$ en papaya Maradol, no encontraron diferencias significativas en la pérdida de peso al utilizar ese recubrimiento en comparación con el testigo, aspecto que coincide con los resultados de esta investigación.

Se ha señalado que el tratamiento de inmersión de la papaya en agua caliente (TH) puede ejercer un efecto directo sobre las ceras epicuticulares, lo que resulta contradictorio a las respuestas observadas en la fruta, pues por un lado autores como Pereira et ál. (2012) encontraron que con el calor, las ceras pueden ser redistribuidas y provocar que la capa que estas forman, quede más delgada lo que da origen a una mayor pérdida de peso mientras que Valero y Serrano (2010) consideran que el tratamiento puede producir un derretimiento de la cera epicuticular, que puede llegar a cubrir las microheridas y estomas, y disminuir con esto la transpiración y la pérdida de peso.

Hasta el momento en que se hicieron las evaluaciones de pérdida de peso ( 4 dsaf), no se observaron diferencias para esa variable entre la fruta del testigo y la que recibió el tratamiento térmico. No obstante, si se toma en consideración la información generada por Vargas (2013), quien encontró que según el mes en que se cosechó la papaya Pococí, la fruta del tratamiento testigo al momento de la madurez perdió entre $3,53 \%$ a $4,72 \%$ del peso original, mientras que en la del tratamiento hidrotérmico esta disminución estuvo comprendida entre 4,11 y $6,02 \%$, lleva a la consideración de la necesidad del encerado en papaya, para evitar que los porcentajes de pérdida de peso sobrepasen el $2 \%$ del peso original, señalado por Nunes y Edmond (2007) como un porcentaje de pérdida de peso crítico a partir del cual se dan los primeros signos de ensuavizamiento. Según esos mismos autores a partir de $4,5 \%$ de pérdida de 
peso, se presenta un ablandamiento externo, cambios en el color y deshidratación que se evidencia como arrugamiento. Las papayas que recibieron el tratamiento con la cera de abejas y aceite de palma, fueron las que presentaron el menor valor de porcentaje de pérdida de peso acumulado, inferior a lo establecido por esos autores como valores limitantes.

Con excepción del tratamiento hidrotérmico (TH) complementado con cera de mezcla de ácidos grasos, se observaron pocos efectos de los tratamientos sobre el desarrollo del color amarillo. En el momento de ingreso al almacenamiento en frío la papaya tenía un $13 \%$ de ese color, el cual continuó incrementándose durante los días que se mantuvo a $12^{\circ} \mathrm{C}$ (Figura 2). El día que finalizó el almacenamiento en frío (dsaf), las frutas de los tratamientos sin cera mostraron una tendencia de más desarrollo de color amarillo. La papaya del tratamiento testigo presentó un $48 \%$ de la superficie de la fruta con ese color, lo que correspondió a un $35 \%$ de aumento de color durante el almacenamiento, situación muy parecida a la de las frutas del tratamiento térmico (TH) sin cera, que mostraron un $45 \%$ de amarillo. La fruta que recibió el tratamiento hidrotérmico complementado $(\mathrm{TH})$ con cera de abejas y aceite de palma presentó durante ese periodo el menor avance de color, un $27 \%$, lo que implicó un $14 \%$ de incremento. Al aumentar la temperatura de almacenamiento a $20^{\circ} \mathrm{C}$, se observó que a los 4 dsaf la fruta de todos los tratamientos, con excepción de la que recibió el tratamiento hidrotérmico complementado con la cera de mezcla de ácidos grasos, continuó su aumento en el porcentaje de color, y la papaya del tratamiento testigo $70 \%$ de amarillo, con valor muy cercano al relacionado por Salazar (2012) respecto a la madurez comercial del híbrido Pococí (75\%) y a partir de esa fecha, no se detectaron diferencias entre las frutas de los distintos tratamientos.

Castricini et ál. (2012) obtuvieron un retraso del color amarillo en papaya Golden luego de utilizar una película de almidón de yuca al $5 \%$, efecto que fue observado en este ensayo y de forma muy evidente a los 4 dsaf solo en las papayas con tratamiento hidrotérmico complementado con la cera de mezcla de ácidos grasos en este ensayo.

Con respecto a las variables de colorimetría $L^{*} a^{*}$ y $b^{*}$ de la cáscara (Figura 3), se encontró diferencias significativas para la

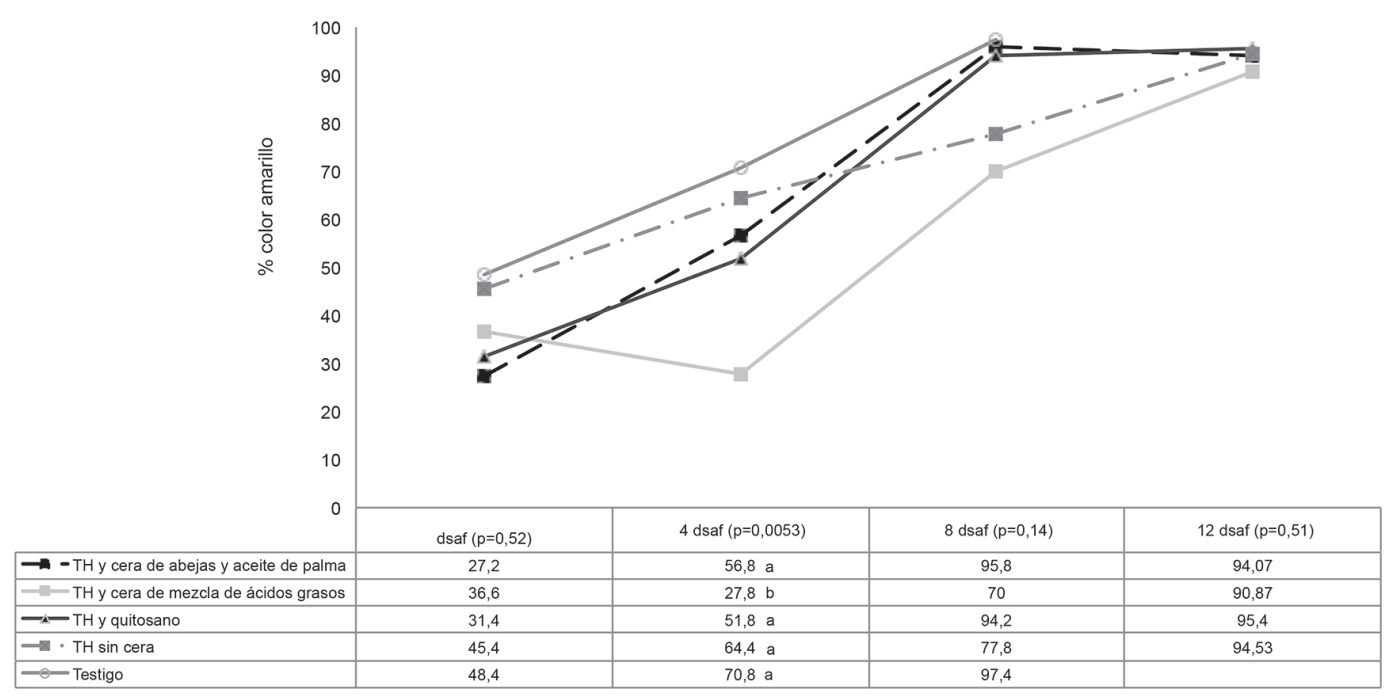

Fig. 2. Avance de amarillo externo en frutas de papaya con tratamiento hidrotérmico $49^{\circ} \mathrm{C} / 20 \mathrm{~min}(\mathrm{TH})$ y ceras. Letras distintas en columnas indican diferencias significativas según prueba LSD Fisher $(\mathrm{p}<=0,05)$. 

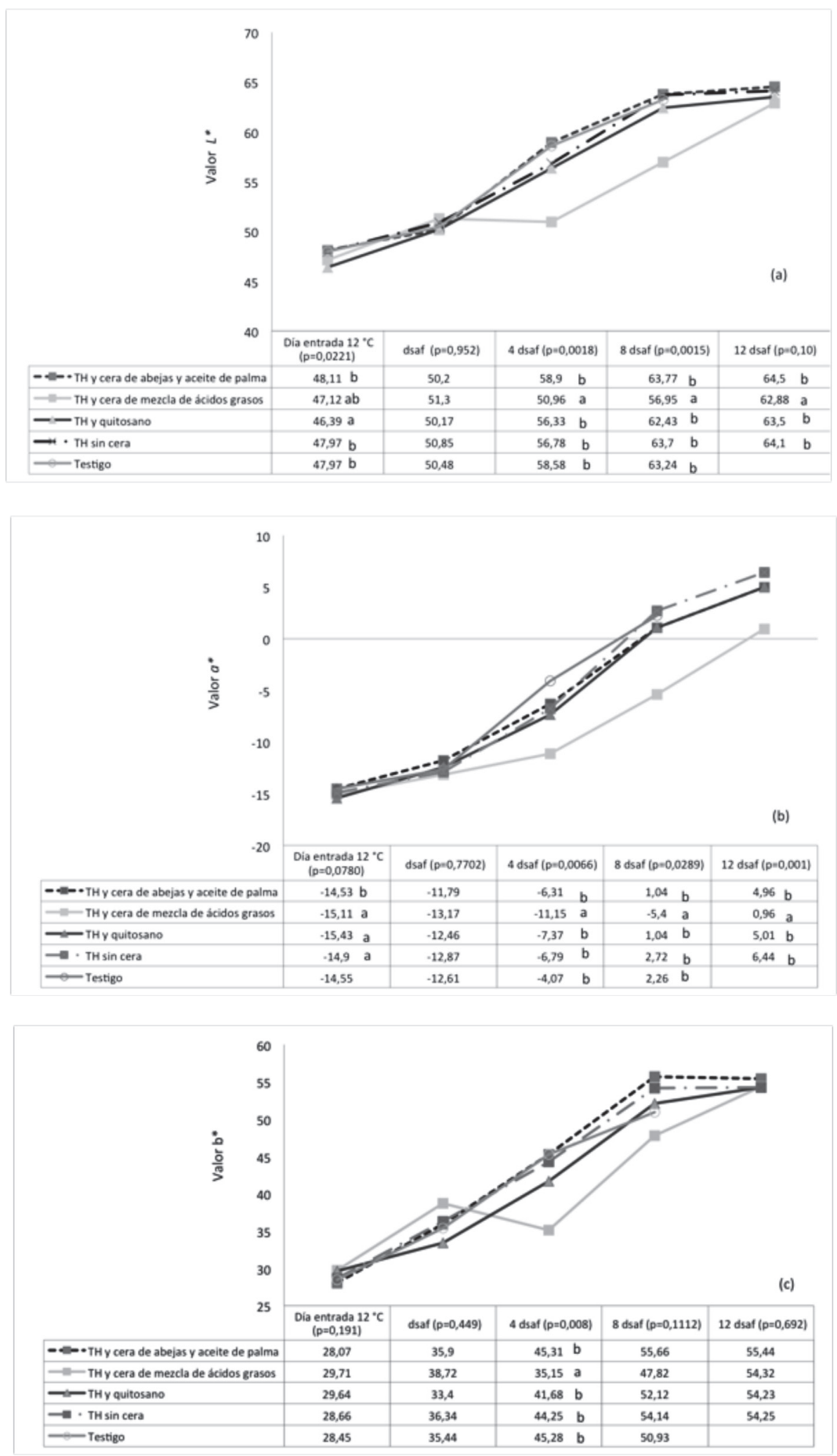

Fig. 3. Variables $\mathrm{L}^{*}, \mathrm{a}^{*}, \mathrm{~b}^{*}(\mathrm{a}, \mathrm{b}, \mathrm{c})$ en frutas de papaya con tratamiento hidrotérmico $49^{\circ} \mathrm{C} / 20 \mathrm{~min}(\mathrm{TH})$ y ceras. Letras distintas en columnas indican diferencias significativas según prueba LSD Fisher $(\mathrm{p}<=0,05)$. 
componente $\mathrm{L}^{*}$ de las papayas a la entrada de cámara, el menor valor lo presentó la fruta tratada con quitosano $0,1 \%$, tratamiento que pudo generar cierta opacidad. A los 4 dsaf y 8 dsaf, la fruta tratada con la mezcla de ácidos grasos presentó menor luminosidad con diferencias estadísticas con respecto al resto de tratamientos. A través de las evaluaciones, las papayas del tratamiento con cera de abejas y aceite de palma, así como las del tratamiento hidrotérmico sin cera y las frutas del tratamiento testigo, no exhibieron diferencias significativas, es decir que las frutas tenían una luminosidad semejante. Lo observado en la fruta que recibió el tratamiento quitosano $0,1 \%$ concuerda con resultados obtenidos en otras investigaciones donde el quitosano $0,75 \%$ disminuyó el valor para $L^{*}$ en papayas de la variedad Eksotika (Eryani et ál. 2008).

En cuanto a la variable $a^{*}$, que mide el avance del color verde hacia el rojo, se encontró que para las fechas 4 dsaf, 8 dsaf y los 12 dsaf, las papayas tratadas con la mezcla de ácidos grasos presentaron los valores más bajos para esta variable, aspecto que refleja un retraso en la degradación de las clorofilas y formación de carotenoides $\mathrm{y}$ antocianinas que son los que dan el color, y que manifiestan en la fruta un predominio de tonalidades más cercanas al color verde, que marca diferencias significativas con respecto al resto de tratamientos. La variable $b^{*}$ exhibió diferencias solamente a los $4 \mathrm{dsf}$, donde las papayas tratadas con ácidos grasos manifestaron un retraso en el avance de los pigmentos que van del azul hacia el amarillo, lo que se percibió en la fruta como un amarillo con menos intensidad en comparación con las papayas de los restantes tratamientos.

En relación con el hue $\left(h^{\circ}\right)$, obtenido a partir de las mediciones de $\mathrm{a}^{*} \mathrm{y} \mathrm{b}^{*}$ en cáscara, tuvo un valor de 112,76 , y a partir desde los 4 dsaf hasta la última fecha de evaluación (12 dsaf), las papayas que recibieron el tratamiento con la cera de mezcla de ácidos grasos $4,7 \%$ tuvieron un hue significativamente mayor con respecto a los demás tratamientos, los cuales no se diferenciaron del testigo, que indica que la fruta tratada con dicha cera retuvo más el color verde que el resto de tratamientos. Este retraso solo afectó el desarrollo de color en la cáscara, ya que no implicó cambios sobre otras variables internas como la tasa respiratoria, el desarrollo de color interno, ni mermó la pérdida de firmeza. Los resultados para el chroma externo $\left(C^{*}\right)$, no evidenciaron diferencias estadísticas excepto a los 4 ddsc (datos no mostrados) donde las papayas tratadas con cera de mezcla de ácidos grasos presentaron la menor intensidad de color con valores que correspondieron a una transición de verde al amarillo opaco y no hubo diferencias importantes entre el resto de tratamientos y el testigo.

Para los valores de L *, a* y b* en pulpa, únicamente hubo diferencias significativas en la fruta que recibió el tratamiento con cera de mezcla de ácidos grasos a los 4 ddsc, la cual presentó los menores valores para $\mathrm{L}^{*} \mathrm{y} \mathrm{b}^{*}$ aunque no implicó un cambio que se mantuviera durante las restantes evaluaciones.

La cera de mezcla de ácidos grasos utilizada en este ensayo también ha sido formulada para ser utilizada en piña pues otorga un mayor brillo a la fruta y retención de color verde (Bai y Plotto 2012), observado también en la papaya al haber inducido el retraso en el desarrollo del color amarillo.

El hue y chroma en pulpa no mostraron diferencias estadísticas que indicaran algún efecto por parte del tratamiento hidrotérmico solo o complementado con las ceras, lo cual permite descartar alguna modificación en el proceso normal de cambios en el color interno con los tratamientos.

La firmeza tanto en cáscara como en pulpa (Cuadro 3), mostraron una pérdida progresiva a partir de la salida del almacenamiento en frío para las diferentes fechas de evaluación, en las que no hubo diferencias estadísticas entre las papayas de los distintos tratamientos, excepto el dsaf y los 12 dsaf, donde las papayas que recibieron encerado con la cera de mezcla de ácidos grasos presentaron una firmeza en cáscara y pulpa significativamente menor en relación con el resto de tratamientos. Los resultados obtenidos por Eryani et ál. (2008), indican que algunas 
Cuadro 3. Firmeza en cáscara, pulpa y sólidos solubles totales en frutas de papaya tratadas con inmersión hidrotérmica $49^{\circ} \mathrm{C} / 20 \mathrm{~min}(\mathrm{TH})$ y ceras.

\begin{tabular}{|c|c|c|c|c|}
\hline \multirow[b]{2}{*}{ Tratamiento } & \multicolumn{4}{|c|}{ Firmeza cáscara $(\mathrm{N})$} \\
\hline & dsaf & 4 dsaf & 8 dsaf & 12 dsaf \\
\hline TH y cera de abejas y aceite de palma & 35,1 & 30,3 & 15,6 & $20,7 \mathrm{~b}$ \\
\hline TH y cera de mezcla de ácidos grasos & 34,1 & 26,1 & 28,4 & $13,5 \mathrm{a}$ \\
\hline TH y quitosano & 38,2 & 32,3 & 22,8 & $21,8 \mathrm{~b}$ \\
\hline TH sin cera & 37,7 & 38,6 & 20,4 & $22,5 \mathrm{~b}$ \\
\hline Testigo & 37,0 & 22,3 & 25,2 & - \\
\hline \multirow[t]{2}{*}{$p$-valor } & 0,54 & 0,87 & 0,36 & 0,0152 \\
\hline & \multicolumn{4}{|c|}{ Firmeza pulpa $(\mathrm{N})$} \\
\hline Tratamiento & dsaf & 4 dsaf & 8 dsaf & 12 dsaf \\
\hline TH y cera de abejas y aceite de palma & $75,6 \mathrm{~b}$ & 44,1 & 30,8 & $28,3 \mathrm{~b}$ \\
\hline TH y cera de mezcla de ácidos grasos & $47,1 \mathrm{a}$ & 39,6 & 25,8 & $12,9 \mathrm{a}$ \\
\hline TH y quitosano & $80,7 \mathrm{~b}$ & 48,5 & 25,0 & $28,8 \mathrm{~b}$ \\
\hline $\mathrm{TH}$ sin cera & $87,0 \mathrm{~b}$ & 64,9 & 25,2 & $33,5 \mathrm{~b}$ \\
\hline Testigo & $79,2 \mathrm{~b}$ & 43,9 & 35,8 & - \\
\hline$p$-valor & 0,0016 & 0,410 & 0,86 & 0,0083 \\
\hline
\end{tabular}

*Letras distintas en columnas indican diferencias significativas según prueba LSD Fisher $(\mathrm{p}<=0,05)$.

coberturas como quitosano al $0,5 \%$ y al $0,75 \%$, incluso combinaciones con calcio, no presentaron diferencias significativas respecto al testigo en cuanto a la firmeza de papaya Eksotika II, por el contrario señalaron que hubo una merma en dicha variable lo que coincide con lo observado en la fruta tratada con ácidos grasos al 4,7\%. Asimismo, Barrera et ál. (2012), no obtuvieron diferencias significativas entre los tratamientos y el testigo luego de aplicar en papaya una cera compuesta por colofonias en relación 1:1 en agua o la combinación de la cera más propóleos al $5 \%$.

En relación con los sólidos solubles totales o grados brix (datos no mostrados), se encontró que la fruta tratada con quitosano $0,1 \%$ registró diferencias al dsaf y mantuvo una tendencia a presentar un mayor contenido de azúcares a lo largo de las evaluaciones. Esta tendencia en el tratamiento quitosano $0,1 \%$ coincide con lo señalado por Ali (2006) y Ali et ál. (2011), quienes relacionaron la aplicación del quitosano al $0,5 \%$ con mayores valores en el contenido de azúcares en comparación con el empleo de las concentraciones al 1\%, 1,5\% y 2,0\%. Su et ál. (2010), citados por Hu et ál. (2011), encontraron que en frutos de litchi que recibieron una cobertura de quitosano, se incrementaron significativamente los niveles de azúcares con respecto al testigo. Almeida et ál. (2011), han señalado que la disminución del contenido de azúcares se puede presentar durante el almacenamiento y se asocia con el autoconsumo de azúcares en la papaya. Esto podría indicar que la fruta tratada con quitosano $0,1 \%$ presentó un proceso de autoconsumo de azúcares más lento que el resto de fruta tratada, aunque no se refleja en la tasa de respiración.

Con respecto a la incidencia de antracnosis y pudrición peduncular, el tratamiento hidrotérmico $(\mathrm{TH})$ redujo significativamente el número de frutas afectadas por esas pudriciones (Figura 4A y Figura 5) en todas las fechas de evaluación, y permitió prolongar por 4 días más la vida útil 

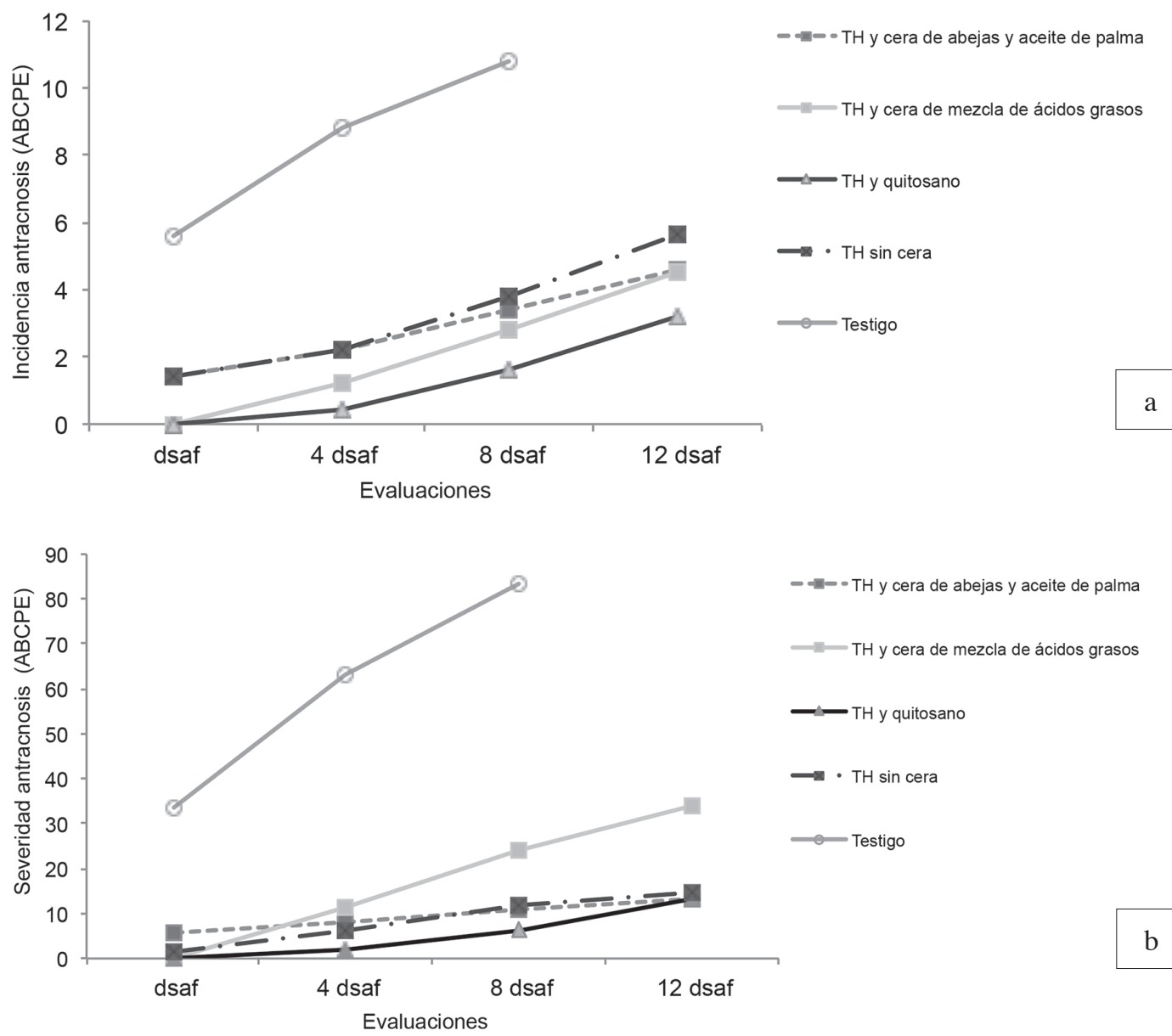

Fig. 4. Desarrollo de antracnosis en frutas de papaya tratadas con tratamiento hidrotérmico $49^{\circ} \mathrm{C} / 20 \mathrm{~min}(\mathrm{TH})$ y ceras. a. Incidencia antracnosis (ABCPE). b. Severidad antracnosis (ABCPE).
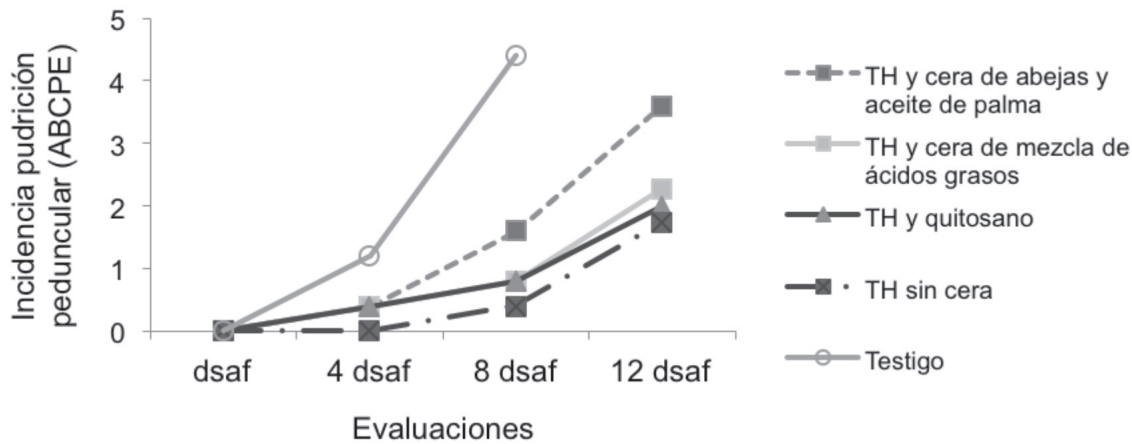

Fig. 5. Incidencia de la pudrición peduncular (ABCPE) en frutas de papaya tratadas con tratamiento hidrotérmico $49^{\circ} \mathrm{C} / 20$ $\min (\mathrm{TH})$ y ceras. 
de la fruta. Umaña et ál. (1993) encontraron resultados similares en el combate de la antracnosis con el tratamiento hidrotérmico en papaya criolla, mientras que Zamora 2012, observó que al someter la papaya híbrido Pococí al tratamiento hidrotérmico a $49^{\circ} \mathrm{C} / 20 \mathrm{~min}$, se retrasó en 3 días la aparición de antracnosis después de la salida de la fruta del almacenamiento en frío. Los tratamientos complementarios con ceras en este ensayo, no confirieron ventajas en la disminución de la incidencia de las pudriciones al compararse con solo el uso del tratamiento $49^{\circ} \mathrm{C} / 20 \mathrm{~min}$, aunque si se observó una tendencia a una menor incidencia y severidad de la antracnosis en la fruta que se sumergió en el quitosano.

En cuanto a la severidad de la antracnosis (Figura 4B), a lo largo de las evaluaciones el tratamiento hidrotérmico $40^{\circ} \mathrm{C} / 20$ min contribuyó a reducir el porcentaje de afectación de la enfermedad en la fruta en comparación con la papaya del tratamiento testigo, sin embargo, el complemento a este tratamiento con ceras no presentó ningún efecto aditivo sobre la severidad de pudriciones. Diferentes estudios han mostrado efectos variables en relación con el efecto de las ceras sobre el control de enfermedades. Al respecto, Berry y Sargent (2004) no encontraron diferencias en cuanto a la severidad de pudriciones en papaya Red Lady luego de aplicar una cobertura a base de aminoetoxi vinil glicina (RETAIN ${ }^{\circledR}$ ), el cual es inhibidor de etileno. Asimismo, Castricini (2009) evaluó en papaya Golden, el efecto de fécula de yuca como barrera protectora y registró en todos los tratamientos pudriciones tanto a nivel de fruta como pedúnculo.

El complementar el tratamiento hidrotérmico $(\mathrm{TH})$ con la cera de abejas y palma, redujo la tasa de respiración de la papaya y la pérdida de peso, sin ningún efecto sobre el desarrollo de color, firmeza de la cáscara y pulpa, grados brix y desarrollo de enfermedades. La respiración es un proceso oxidativo de las sustancias de reserva en la papaya, que conduce a la senescencia pero que al usar ceras se logra disminuir la tasa respiratoria, lo que permite mantener una mejor apariencia de la papaya. Dada la variedad de formulaciones de ceras disponibles en el mercado, es importante continuar con la valoración de nuevas ceras, para seleccionar aquellas que consideren en su manufactura las características de la papaya y las características de los consumidores en los distintos mercados donde se comercializa papaya.

\section{AGRADECIMIENTOS}

Esta investigación fue realizada con fondos de Fittacori del Ministerio de Agricultura y Ganadería y de la Vicerrectoría de Investigación de la Universidad de Costa Rica, en colaboración con Centro Agrícola Cantonal de Guácimo que aportó la fruta.

\section{LITERATURA CITADA}

ACOSTA M., NIETO D., DOMÍNGUEZ J.L., DELGADILLO F. 2001. Calidad y tolerancia en frutos de papaya (Carica papaya L.) a la inoculación del hongo Colletotrichum gloeosporioides Penz., en postcosecha. Chapingo. Serie Horticultura 7(1):119130.

ALI A. 2006. Antracnose incidence, biochemical change, postharvest quality and gas exchange of chitosan coated-papaya. (Abstract) Tesis Ph. D Putra, MY. Universiti Putra $20 \mathrm{p}$.

ALI A., MAHMUD T.M.M., KAMARUZAMAN S. 2011. Effect of chitosan coatings on the physicochemical characteristics of Eksotika II papaya (Carica papaya L.) fruit during cold storage. Food Chemistry 124:620-626.

ALMEIDA A., REIS J.D., SANTOS D., VIEIRA T., DA COSTA M. 2011. Estudio de la conservación de la papaya (Carica papaya L.) asociado a la aplicación de películas comestibles. Revista Venezolana de Ciencia y Tecnología de Alimentos 2:49-60.

ARAUZ L.F., NAVARRO J.R. 1999. Exactitud y repetibilidad de dos métodos para la evaluación de la severidad de enfermedades fungosas en el fruto de papaya (Carica papaya L.). Agronomía Costarricense 23(1):89-96.

BAI J., PLOTTO A. 2012. Coatings for fresh fruits and vegetables, pp. 185-242. In: E.A Baldwin, R. Hagenmaier and J. Bai (eds.). Edible coatings and films to improved food quality. Second edition. Taylor \& Francis Group, United States.

BARTZ J.A., BRECHT J.K. 2003. Postharvest physiology and pathology of vegetables. 2 ed. Andexp. Florida, US, Marcel Dekker. 705 p.

BARRERA E.B., GIL M.L., GARCÍA C.P., DURANGO L.R., GIL J.G. 2012. Empleo de un recubrimiento 
formulado con propóleos para el manejo poscosecha de frutos de papaya (Carica papaya L.cv. Hawaiana). Revista Facultad de Ciencias Agrarias de Medellín de Colombia 65:6496-6506.

BAUTISTA B.S., HERNÁNDEZ M., BOSQUEZ M., WILSON C.L. 2003. Effects of chitosan and plant extracts on growth of Colletotrichum gloeosporioides, anthracnose levels and quality of papaya fruit. Crop Protection 22:1087-1092.

BAUTISTA S., HERNÁNDEZ A.N., VELÁZQUEZ DEL VALLE M.G., HERNÁNDEZ M., AIT E., BOSQUEZ E., WILSON C.L. 2006. Chitosan as a potential natural compound to control pre and postharvest diseases of horticultural commodities. Crop Protection 26:108-118.

BEIRUTH L.J., DE SOUZA M.L., DE ARÁUJO S.E., MORÁIS A.P. 2009. Resvestimentos alternaivo na conservação pos-colheita de maracujá amárelo. Revista Brasileira de Fruticultura Jaboticabal 31(4):995-1004.

BERRY A.D., SARGENT S.A. 2004. Pre and postharvest application of Retain ${ }^{\circledR}$ to Red Lady papaya fruit: effects on harvest maturity, ripening and quality. Proceedings Florida State Horticultural Society 117:389-391.

CASTRICINI A. 2009. Aplicação de Revestimentos comestíveis para conservação de mamões (Carica papaya L.) Golden. Tesis Ph.D., UFRRJ, Rio de Janeiro, Br. 106 p.

CASTRICINI A., CONEGLIAN R.C., DELIZA R. 2012. Revestimento de mamão papaia por películas de amido: efeito nas características sensoriais. Ciência e Tecnologia de Alimentos Campinas 32:84-92.

ERYANI A.R., MAHMUD T.M., OMAR S.R., ZAKI A.R. 2008. Effects of calcium infiltration and chitosan coating on storage life and quality characteristics during storage of papaya (Carica papaya L.). International Journal of Agricultural Reserch 3(4):296-306.

FALLIK E. 2004. Prestorage hot water treatments (immersion, rising and brushing). Postharvest Biology and Technology 32:125-134.

FAO (ORGANIZACIÓN DE LAS NACIONES UNIDAS PARA LA AGRICULTURA Y LA ALIMENTACIÓN,IT). 2013.La producción mundial de fruta tropical alcanzará 82 millones de toneladas en 2014 Roma, IT. Consultado: 15 marzo 2013. Disponible en http://www.fao.org/agronoticias/agronoticias/detalle/es/?dyna_fef\%5Buid\%5D=159358

GONZÁLEZ E., UMAÑA G., ARAUZ L.F. 1999. Combate de la pudrición peduncular del mango causada por Botryodiplodia theobromae Pat. mediante el mantenimiento de los pedicelos y el deslechado sobre láminas. Agronomía Costarricense 23:31-35.

HU H., LI X., DONG C., CHEN W. 2011. Effects of wax treatment on quality and postharvest physiology of pineapple fruit in cold storage African Journal of Biotechnology 10(39):7592-7603.

LARA I., BELGE B., GOULAO L.F. 2014. The fruit cuticle as a modulator of postharvest quality. Postharvest Biology and Technology 87:103-112.

LURIE S. 1998. Postharvest heat treatments: review. Postharvest Biology and Technology 14:257-269.

LURIE S., MITCHAM E.J. 2007. Physiological responses of agriculture commodities to heat treatments, pp. 79-104. In: J. Tang, E. Mitchan, S. Wang and S. Lurie (eds.). Heat treatments for postharvest pest control: theory and practice. CAB International, United States.

NUÑEZ C.N., EMOND J.P. 2007. Relationship between weight loss and visual quality of fruits and vegetables. Proceedings of the Florida State Horticultural Society 120:235-245.

PAULL E.R., CHEN J.N. 1983. Postharvest variation in cell wall degrading enzymes of papaya (Carica papaya L.) during fruit ripeniing. Plant Physiology 72:382385 .

PEREIRA C., SHERRER C.R., ZAPATA C.P., TESSARO I.C., FERREIRA L., BENDER R. 2012. Effects of ozone in combination with hydrothermal treatment and wax on physical and chemical properties of papayas. Science and Engineering 34:1-7.

PROCOMER. 2014. Portal Estadístico de Comercio Exterior. San José, Costa Rica. Consultada en febrero 2015. Disponible en http://servicios.procomer.go.cr/ estadisticas/inicio.aspx

PROTSENKO M.A., BULANTSEVA E.A., KORABLEVA N.P. 2010. Polygalacturonase inhibiting proteins in plant fleshy fruits during their ripening and infections. Russian Journal of Plant Physiology 57(3):356-362.

SABORÍO A. 2012. Plan de trabajo para el programa de certificación de papaya (Diapositivas). San José, CR. 17 diapositivas, color (Disponible en CD ROM la memoria del curso manejo y perspectivas del cultivo de papaya (Carica papaya L.).

SALAZAR D. 2012. Establecimiento de la escala de madurez y potencial de vida útil durante el almacenamiento de papaya (Carica papaya L.) híbrido Pococí. Tesis de licenciatura, Universidad de Costa Rica, San José, Costa Rica. 91 p.

TANG J., MITCHAM E., WANG S., LURIE E. 2007. Heat treatment for postharvest pest control: theory and practice. Massachusetts, US, Cromwell Press. 349 p.

UMAÑA G., ARAUZ L.F., SABORÍO D. 1993. Combate de Antracnosis en papaya criolla I. Tratamientos poscosecha químicos y térmicos. En: Resúmenes de Trabajos Científicos IX Congreso Nacional Agronómico y de Recursos Naturales. San José, CR. 18-22 de octubre, Volumen II (2):137.

UMAÑA G., LORÍA C.L., GÓMEZ J.C. 2011. Efecto del grado de madurez y las condiciones de 
almacenamiento sobre las características fisicoquímicas de la papaya híbrido Pococí. Agronomía Costarricense 32(2):61-73.

VALENCIA S.A., PÉREZ M.B., DEL RIO M.A., PALOU L. 2009. Effect of antifungal hydroxypropyl methylcellulose (HPMC)-lipid edible composite coatings on postharvest decay development and quality attributes of cold-stored 'Valencia' oranges. Postharvest Biology and Technology 54:72-79.

VALERO D., SERRANO M. 2010. Postharvest biology and technology for preserving fruit quality. CRC Press, United States. $255 \mathrm{p}$.
VARGAS T. 2013. Ultraestructura y calidad asociadas a época de cosecha y manejo poscosecha en frutos de papaya (Carica papaya L.) híbrido Pococí. Tesis de licenciatura, Universidad de Costa Rica, San José, Costa Rica. 92 p.

ZAMORA J.E. 2012. Control de antracnosis (Colletotrichum gloeosporioides Penz.) en frutos de papaya (Carica papaya L.) cv. Pococí mediante aplicaciones poscosecha de agua caliente y cloruro de calcio. Tesis de licenciatura, Universidad de Costa Rica, San José, Costa Rica. 103 p. 
